\title{
Repercusión de la frigoconservación de la aceituna de molino en el proceso de producción de aceite de oliva virgen
}

\author{
Por Miguel Canet ${ }^{1}$ y José M. ${ }^{a}$ García ${ }^{2}$ \\ 1 Cooperativa Comarcal «Valle de Albaida», Ctra. Xátiva, s/n. 46837 - Quatretonda, Valencia. \\ 2 Instituto de la Grasa (CSIC), Avda. Padre García Tejero, 4. 41012 - Sevilla.
}

\section{RESUMEN}

Repercusión de la frigoconservación de la aceituna de molino en el proceso de producción de aceite de oliva virgen.

Durante la campaña 1993-94, un total de $1.003 .351 \mathrm{Kg}$. de aceitunas (Olea europaea, cvs. Blanqueta y Villalonga) fueron almacenadas en una cámara frigorífica a $5^{\circ} \mathrm{C}$ por un tiempo máximo de 18 días. El aceite virgen obtenido de estas aceitunas mostraba unos índices físico-químicos y una valoración sensorial dentro de los límites establecidos para el aceite de oliva virgen "extra". La frigoconservación de la aceituna hizo posible el trabajo a máximo rendimiento de la maquinaria de procesamiento durante 8 semanas, sin que se redujera apreciablemente la calidad del aceite virgen obtenido. Además, permite una mejor amortización de los equipos disponibles y, al requerir que el fruto se distribuya en cajas, contribuye en la racionalización de la manipulación postcosecha de la aceituna.

PALABRAS-CLAVE: Aceituna - Almacenamiento - Olea europaea - Postcosecha - Refrigeración.

\section{SUMMARY}

Repercussion of the mill olive cool-storage on the production process of virgin olive oil.

During the $1993-94$ season, a total of $1,003,351 \mathrm{Kg}$. of olive fruits (Olea europaea, cvs. Blanqueta y Villalonga) were stored in a cool room at $5{ }^{\circ} \mathrm{C}$ until a maximum time of 18 days. The virgin olive oil obtained from these fruits showed physical, chemical and sensorial indices inside the limits established for the "extra" virgin olive oil. The cool-storage of the olive fruits made possible a maximum yield in the processing machinery for 8 weeks without an appreciable lost of quality on the virgin olive oil obtained. It allows a better amortisation of the disposable equipments and, requiring the distribution of the fruit in boxes, contributes to the rationalisation of the olive fruit postharvest manipulation. Storage.

KEY-WORDS: Olea europaea - Olive - Postharvest - Refrigeration -

\section{INTRODUCCIÓN}

La calidad del aceite virgen de oliva está en relación directa con la fisiología de la aceituna de donde es extraído, a menos que haya sufrido un deterioro posterior. Algunos factores previos a la cosecha pueden afectar al fruto (climatología, irrigación, plagas, carga de fruto en el árbol, etc.) (Barone et al., 1994). Sin embargo, las alteraciones más importantes de la fisiología del fruto tienen lugar durante el tiempo que media entre la cosecha del fruto y su procesamiento. La enorme masa de aceitunas, más de 2 millones de toneladas, que se cosecha anualmente para la producción de aceite en España no puede ser procesada de inmediato por la industria almazarera (Maté, 1996). En consecuencia, la aceituna necesita ser almacenada. Las grandes diferencias de producción de un año a otro hacen muy variable, tanto el tiempo de almacenamiento, como la carga que se debe almacenar, pudiendo variar desde 4 a 5 días y menos de $1.000 \mathrm{Kg}$ hasta varios meses y más de 1.000 .000 de Kg (Kiritsakis, 1991). La conservación de estas aceitunas se ha venido realizando tradicionalmente mediante amontonamiento en pilas, que vulgarmente se han denominado «trojes". La aceituna así conservada sufre en corto tiempo importantes alteraciones. EI apelmazamiento provoca la destrucción física de los frutos, especialmente los localizados en la zona inferior, que soportan todo el peso del conjunto, produciéndose una liberación de material orgánico, excelente caldo de cultivo para la proliferación de hongos y bacterias saprofíticos. En estas condiciones se desarrollan diferentes procesos degenerativos: anaerobios principalmente en la zona interna del troje y aerobios en la externa. Al mismo tiempo, estos fenómenos originan una elevación notable de la temperatura que, a su vez, acelera autocatalíticamente todo el proceso degradativo. En cuestión de horas se produce una descomposición generalizada de las estructuras orgánicas de la aceituna y una alteración de sus componentes químicos. No es, pues, de extrañar, que el aceite obtenido a partir de estos frutos no sea apto para el consumo sin una refinación previa. Esto implica un gasto de procesamiento adicional a la pérdida del valor intrínseco de la calidad del aceite. Los aceites atrojados presentan, además de una alta acidez y una baja estabilidad, una cantidad anormalmente alta de ácidos 
volátiles, como acético o butírico y de alcoholes grasos, como 1-octen-3ol y 2-octen-1ol, siendo la asociación de estos compuestos la causa principal del aroma desagradable peculiar que los caracteriza (Gutiérrez et al., 1981, Olías et al., 1988).

El Departamento de Fisiología y Tecnología de Productos Vegetales del Instituto de la Grasa ha estudiado a escala de laboratorio las condiciones idóneas de almacenamiento de la aceituna de molino para la obtención de un aceite de calidad (Pérez-Camino et al., 1992; Gutiérrez et al., 1992; García, 1993; García et al., 1994; García et al., 1996). En estos trabajos se puso de manifiesto que la refrigeración a $5{ }^{\circ} \mathrm{C}$ de la aceituna «Picual» en envases plásticos con capacidad para $60 \mathrm{Kg}$ de fruto permitía durante más de 30 días la obtención de aceite de oliva virgen extra.

La Cooperativa Comarcal del Valle de Albaida está formada por 17 cooperativas de productores que agrupan un total de 2.200 olivicultores con una

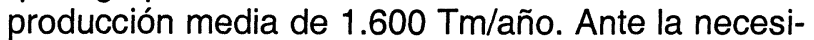
dad de dar respuesta a un aumento de producción que excedería su capacidad de molturación durante la campaña 1993-94, se llevó a cabo la conservación a $5^{\circ} \mathrm{C}$ del fruto que no pudo ser inmediatamente procesado. En el presente trabajo se presentan los resultados obtenidos en la citada campaña.

\section{PARTE EXPERIMENTAL}

Un total de $1.003 .351 \mathrm{Kg}$ de aceitunas (Olea europaea, cvs. Blanqueta y Villalonga) fueron cosechadas a mano por "ordeño» con una media de grado 3 de maduración (Uceda y Frías, 1975) entre Noviembre de 1993 y Enero de 1994 en la comarca del Valle de Albaida (Valencia). Estos frutos fueron envasados en contenedores plásticos capaces para $14 \mathrm{Kg}$ y almacenados en una cámara frigorífica a $5{ }^{\circ} \mathrm{C}$ por un tiempo máximo de 18 días (tiempo medio 14 días y 5 horas). Posteriormente el fruto fue procesado en un sistema de tres fases a razón de $32.000 \mathrm{Kg} /$ día. Tres muestras de aceites, obtenidas en diferentes fechas, entre la sexta y la novena semanas de campaña (período de la campaña en que el volumen de aceituna almacenada alcanzó el máximo y, en consecuencia, la demora en el procesamiento fue mayor) fueron analizadas considerando los parámetros siguientes: Los índices físico-químicos que evalúan su calidad (acidez, índice de peróxidos y coeficientes de extinción específica a 232 y $270 \mathrm{~nm}$ ) de acuerdo con las normas UNE (AENOR, 1973a-c). La estabilidad ante la oxidación fue medida por el método Rancimat (Läubli y Bruttel, 1986; Gutiérrez, 1989). La calidad sensorial fue evaluada por un panel analítico de 12 miembros, según el método descrito en el Anexo XII de las Reglas de la Comunidad Economica Europea (2568/91). El aceite fue valorado en una escala de 9 puntos, siendo 1 la puntuación peor posible y 9 la correspondiente a una calidad óptima. Los resultados de estos parámetros corresponden a los valores medios \pm su desviación típica de las tres tomas de muestra realizadas.

\section{RESULTADOS Y DISCUSIÓN}

La calidad de los aceites vírgenes obtenidos a partir de la aceituna conservada a $5{ }^{\circ} \mathrm{C}$ se mantuvo durante 18 días dentro de la categoría "extra» en todos los parámetros analizados (Tabla I), presentando además valores de desviación típica muy bajos. Los valores de acidez son los que presentan un mayor grado de variabilidad, pero las desviaciones típicas de este parámetro no llegan a superar el $50 \%$ de los valores medios en los aceites de las dos variedades analizadas y sumadas a éstos aun están lejos de aproximarse al valor del $1 \%$, considerado como límite máximo para los aceites vírgenes de calidad "extra».

Tabla I

Evaluaciones alcanzadas por los aceites obtenidos a partir de aceitunas de las variedades Blanqueta y Villalonga almacenadas industrialmente durante 18 días a $5^{\circ} \mathrm{C}$ en los parámetros que definen la calidad del aceite de oliva virgen $\left(^{*}\right)$

\begin{tabular}{lcc}
\hline \multicolumn{1}{c}{ Parámetro } & Blanqueta & Villalonga \\
\hline Acidez (\% ácido oleico) & $0,53 \pm 0,19$ & $0,44 \pm 0,12$ \\
Índice de peróxidos (mEq Oxígeno/Kg aceite) & $3,50 \pm 0,65$ & $3,87 \pm 0,98$ \\
$K_{232}$ & $1,68 \pm 0,06$ & $1,57 \pm 0,09$ \\
$K_{270}$ & $0,12 \pm 0,00$ & $0,14 \pm 0,02$ \\
Estabilidad (horas) & $51,13 \pm 6,96$ & $67,67 \pm 9,59$ \\
Valoración sensorial ("1" pésimo - "9" óptimo) & $6,93 \pm 0,37$ & $7,30 \pm 0,22$ \\
\hline
\end{tabular}

Cada cifra corresponde al valor medio \pm la desviación típica de aceites procedentes de tres extracciones diferentes.

El almacenamiento a $5{ }^{\circ} \mathrm{C}$ de la aceituna que no pudo ser procesada inmediatamente entre la quinta y la séptima semanas de campaña, permitió que la maquinaria extractora fuera utilizada a pleno rendimiento durante 8 semanas, sin que se produjera un deterioro de la calidad del aceite (Fig. 1).

$\mathrm{Si}$, redondeando cifras, se considera el total almacenado como de $1000 \mathrm{Tm}$ de aceitunas, el aceite obtenido puede estimarse en $200 \mathrm{Tm}$ de calidad virgen "extra» con un rendimiento en la extracción del $20 \%$. En el supuesto de que se hubiera dejado atrojar la aceituna, se puede estimar que, como máximo, un $20 \%$ del total de este aceite conserva un grado de calidad que no hace necesaria su refinación. En consecuencia, mediante la conservación en frío de la aceituna se puede conseguir, empleando las mis- 
mas condiciones de trabajo de la Cooperativa del Valle de Albaida, por cada $1000 \mathrm{Tm}$ de aceituna una producción mínima aproximada de $160 \mathrm{Tm}$ de aceite virgen comercializable más que si se atrojara. Según la referencia de precios de mercado de Maté (1997) durante el período entre Abril y Junio la diferencia de precio media entre el oliva virgen y el oliva virgen refinable fue 84,4 ptas $/ \mathrm{Kg}$. Por tanto, sin tener en cuenta que el aceite producido fue de calidad "extra» y considerándolo simplemente como virgen comercializable sin refinación, la conservación en frío supone un beneficio potencial bruto de aproximadamente de 13.504.000 ptas por cada $1000 \mathrm{Tm}$ de aceituna conservada a $5^{\circ} \mathrm{C}$. Para estimar el beneficio potencial neto a este valor se le debe sustraer el incremento de gasto que supone el uso de la refrigeración. En el caso de la Cooperativa del Valle de Albaida, que posee cámaras de refrigeración para cítricos, este gasto se ha evaluado, incluyendo el alquiler de las cajas necesarias para el almacenamiento de la aceituna, en 2,1 ptas./Kg. Además permite una mejor amortización de la inversión realizada en las cámaras, pues la conservación de aceitunas y cítricos es perfectamente compatible, dado que no coinciden en la misma época del año.

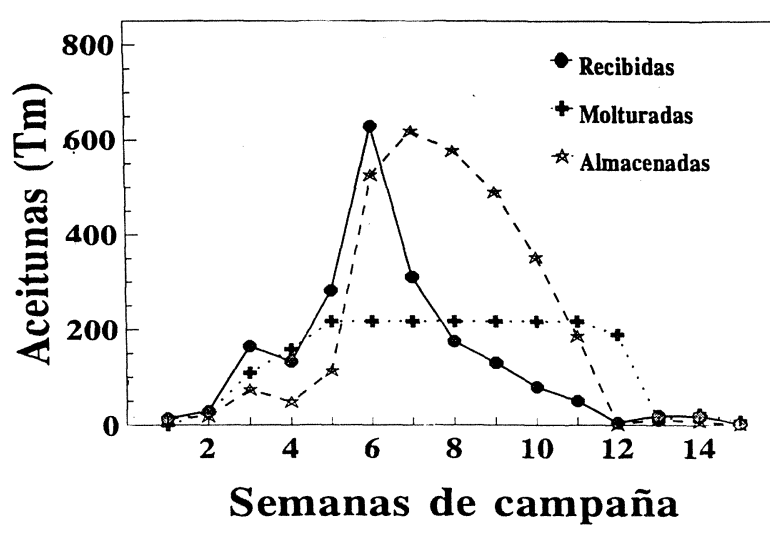

Figura 1

Datos de la evolución de la campaña 1993-94 en el procesamiento de aceituna para la obtención de aceite de oliva virgen con una capacidad de molturación de $32.000 \mathrm{~kg} / \mathrm{día}$ en la Cooperativa Comarcal del Valle de Albaida. El fruto se conservó a $5^{\circ} \mathrm{C}$ en envases capaces para $14 \mathrm{Kg}$.

Si como solución alternativa se hubiera doblado la capacidad de procesamiento hasta un total de $64.000 \mathrm{Kg} . /$ día (Fig. 2), este aumento por sí solo no habría sido suficiente para absorber en su totalidad el pico máximo de producción, dejando un remanente de más de $200 \mathrm{Tm}$ de fruta sin procesar en la sexta semana de campaña. Esta aceituna necesita al menos tres días más para ser procesada, tiempo en el que, según las condiciones de almacenamiento, puede deteriorarse y paralelamente sufrir una degradación en la calidad del aceite. Por otra parte, el tiempo en que el equipo pudiera trabajar a pleno rendimiento se limitaría a sólo dos semanas, lo que representa apenas un $13,3 \%$ del total de la campaña.

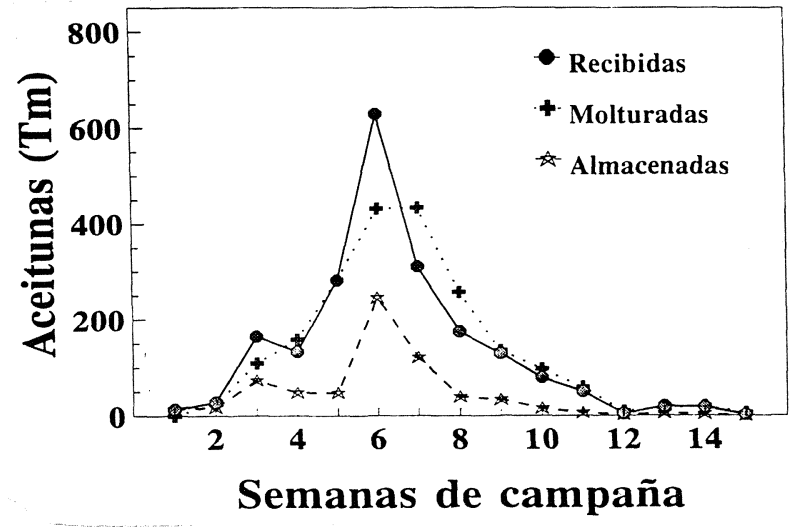

Figura 2

Datos estimados de la evolución de la campaña 1993-94 en el procesamiento de aceituna para la obtención de aceite de oliva virgen bajo el supuesto del uso de una capacidad de molturación de $64.000 \mathrm{Kg} / \mathrm{día}$ en la Cooperativa Comarcal del Valle de Albaida.

Otro factor que debe tenerse en cuenta es la alternancia característica de la aceituna. Si se considera una campaña en la que la producción sea exactamente la mitad de la estudiada en este trabajo (Fig. 3), la capacidad de molturación existente (32.000 $\mathrm{Kg} . / \mathrm{di} a$ ) habría sido suficiente para procesar todo el material recibido sin necesidad de conservar o de incrementar la capacidad de procesamiento.

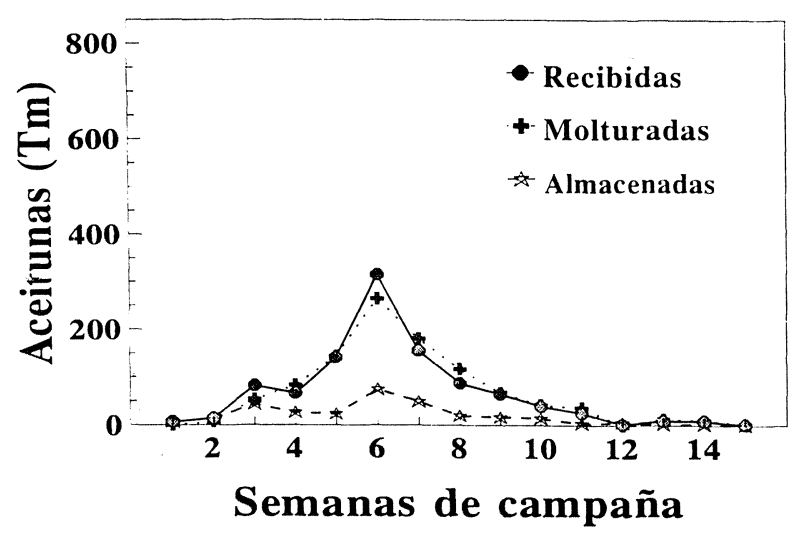

Figura 3

Datos estimados de la evolución de una campaña de procesamiento de aceituna para la obtención de aceite de oliva virgen bajo el supuesto de una reducción del $50 \%$ en el aporte de fruto, tomándose como referencia la campaña 1993-94

Otra ventaja de la conservación refrigerada es que, en este caso, puede ser contratada mediante arrendamiento más fácilmente que la capacidad de procesamiento de aceituna. Cuando se produce el máximo de producción todas las almazaras de una misma zona están trabajando a pleno rendimiento, mientras que las cámaras frigoríficas pueden estar asequibles, a menos que ya se estén empleando 
para conservar aceituna. En cambio el aumento de la capacidad de molturación supone la compra de un nuevo equipo de lenta amortización y utilizable sólo para la obtención de aceite. La refrigeración, aparte de ser más versátil, puesto que se puede emplear para la conservación de otros productos además de la aceituna, permite una mejor amortización de la maquinaria molturadora disponible y aumenta de hecho la capacidad real de procesamiento sin necesidad de incrementar el número de líneas de molturación y sin que se corra el riesgo de un deterioro del fruto y del aceite que éste contiene. Enfocado desde un punto de vista de futuro, el uso de la refrigeración supone un paso importante en la racionalización del proceso de producción del aceite de oliva. Al requerir el uso de cajas para el manejo del fruto, obliga al industrial a tratar a la aceituna con la consideración que merecen otros frutos. Con ello se facilita su clasificado según: variedad, lugar de procedencia, grado de maduración, grado de humedad, tiempo de demora, cosecha en suelo o en árbol, etc., para proceder a su molturación en un orden lógico, agrupando o separando las partidas según su calidad o como más interese. Además, permite el uso de los sistemas paletizados para transporte, almacén, lavado, etc., utilizados en la manipulación postcosecha. Esta racionalización, aunque conlleve un inevitable coste, es ineludible si se desea mejorar el nivel de calidad de los aceites vírgenes de oliva, ya que la principal característica comercial que puede hacer competitivos a estos aceites frente a los de semillas es el desarrollo al máximo de su calidad (Luchetti, 1993).

Acometer la construcción de una cámara con 75 C.V. de potencia capaz de almacenar $500.000 \mathrm{Kg}$. de aceitunas con una capacidad de recepción de $20.000 \mathrm{Kg}$./hora y dotada de un túnel de preenfriado, que permite la reducción rápida de la temperatura del fruto e incluyendo la construcción del edificio, la instalación y las puertas, supondría una inversión aproximada a los 30 millones de pesetas.

\section{BIBLIOGRAFÍA}

AENOR (1973a).— «Cuerpos grasos. Determinación de la acidez libre".-Norma española. UNE 55011-73; Asociación Española de Normalización y Certificación, Madrid.

AENOR (1973b).- «Materias grasas. Índice de peróxidos".Norma española. UNE 55023-73; Asociación Española de Normalización y Certificación. Madrid.
AENOR (1973c).—Materias grasas. Medidas espectrofotométricas de la absorción en la región ultravioleta".-Norma española. UNE 55047-73; Asociación Española de Normalización y Certificación, Madrid.

Barone, E. Gullo, G., Zappia, R. y Inglese, P. (1994).«Effects of crop load on fruit ripening and olive oil quality".-J. Hort. Sci. 69, 67-73.

García, J.M. (1993)._- Efecto del $\mathrm{CO}_{2}$ en la atmósfera de almacenamiento del fruto sobre la calidad del aceite de oliva".-Grasas y Aceites. 44, 169-174.

García, J.M., Gutiérrez, F., Castellano, J.M., Perdiguero, S., Morilla, A. and Albi, M.A. (1994). - «Storage of olive destined for oil extraction".-Acta Horticulturae, 368, 673-681.

García, J. M., Gutiérrez, F., Castellano, J. M., Perdiguero S., Morilla, A. and Albi, M. A. (1996).- «Influence of storage temperature on fruit ripening and olive oil quality".-J. Agric. Food Chem., 44, 264-267.

Gutiérrez, R., Dobarganes, M. C., Gutiérrez, F. y Olías, J. M. (1981).- «Componentes volátiles en el aroma del aceite de oliva virgen. V. Aceites obtenidos de frutos atrojados".-Grasas y Aceites, 32, 299-303.

Gutiérrez, F. (1989).— - ¿Determination of oxidative stability in virgin olive oils: comparison between active oxygen method and Rancimat method".-Grasas y Aceites, 40, 1-5.

Gutiérrez, F., Perdiguero, S., García, J. M. and Castellano, J. M. (1992). - «Quality of oils from olives stored under controlled atmosphere».-J. Am. Oil Chem. Soc. 69, 1215-1218.

Kiritsakis, A. K. (1991).-Editor. «Olive Oil».192 p.-American Oil Chemist'Society. Champaign, Illinois. USA.

Läubli, W. y Bruttel, P. A. (1986).- «Determination of the oxidative stability of fats and oils by the Rancimat method".-J. Am. Oil Chem. Soc. 63, 792-794.

Luchetti, F. (1993). - «El COI y la mejora de calidad».Agricultura, 730, 384-385.

Maté, V. (1996).— «Memorándum sobre el sector del aceite de oliva".-Dossier Óleo 3. ${ }^{\text {er }}$ trimestre, 44-61.

Maté, V. (1997).- «Evolución de precios de aceites de oliva y orujo".-Dossier Óleo $2{ }^{\circ}$ trimestre, 20-21.

Olías, J. M., Lozano, M. D., Ríos, J. J., Gutiérrez, F. y Gutiérrez, R. (1988).- «Determinación de ácidos grasos volátiles en aceites de oliva vírgenes».Grasas y Aceites, 39, 77-81.

Pérez-Camino, M. C., García, J. M., Castellano, J. M. (1992).«Polar compound concentration in virgin oils from stored cultivar Picual olive olives".-J. Agric. Food Chem., 40, 2260-2262.

Uceda, M. y Frías, L. (1975).— «Fechas de cosecha. Evolución del contenido en aceite del fruto, la composición y la calidad del aceite".-En las Actas de II Seminario Oleícola Internacional. C.O.I.: Córdoba, España, pp. 125-130. 